\title{
PARASITEMIA EM PACIENTES CHAGÁSICOS CRÔNICOS AVALIADA PELO ÍNDICE DE TRIATOMÍNEOS INFECTADOS NO XENODIAGNÓSTICO.
}

\author{
José Borges Pereira1, Henry Percy Faraco Willcox ${ }^{1}$, Carlos Brisola Marcondes ${ }^{2}$ \\ e José Rodrigues Coura1.
}

\begin{abstract}
Como parte de um estudo pré-terapêutico da infecção chagásica crônica, a parasitemia de 206 pacientes ( 85 homens e 121 mulheres, com idades de 7 a 80 anos, média de 37,4 $\pm 14,6$ ) do municipio de Virgem da Lapa, Minas Gerais, foi avaliada pelo índice de ninfas de triatomíneos infectadas nos xenodiagnósticos aplicados. Cada paciente foi submetido a três exames no período de 12 meses. Em cada exame foram aplicadas 40 ninfas de $3 \%$ ou $4 .^{\circ}$ estádios de Triatoma infestans $A$ parasitemia de cada paciente foi classificada arbitrariamente, de acordo com o percentual de ninfas positivas no total de examinadas nos três xenos, em: não detectada - todas as ninfas negativas; baixa - quando o valor era maior que zero ou até igual a 2,0\%; média-quando maior que $2,0 \%$ ou até igual a 7,0\%; $e$ alta-quando era maior que 7,0\%. Com esses critérios a parasitemia não foi detectada em 105 (51,0\%) pacientes, foi baixa em 55 (26,7\%), média em $27(13,1 \%)$ e alta em 19 (9,2\%). Não houve diferença nos níveis da parasitemia em relação ao sexo dos pacientes, mas em relação aos grupos etários foi maior a freqüencia da parasitemia baixa no grupo de 60 ou mais anos. A parasitemia alta foi mais freqüente no grupo de pacientes com cardiopatia crônica chagásica. $A$ "parasitemia persistente" (todos os xenopositivos) foi observada em todos os pacientes com parasitemia alta, em $22,2 \%$ dos que apresentaram parasitemia média e em nenhum com parasitemia baixa.
\end{abstract}

Palavras-chaves: Doença de Chagas. Xenodiagnóstico. Parasitemia. Triatoma infestans.

A pesquisa do Trypanosoma cruzi na corrente sangüinea de pacientes chagásicos crônicos tem sido realizada, especialmente, através de métodos indiretos como xenodiagnóstico, hemocultura e inoculação em animais sensíveis. Esses métodos, embora apresentem baixo rendimento 112182627 , têm se mostrado mais eficientes do que o exame direto do sangue 2324 .

A medida da parasitemia em pacientes chagásicos crônicos não dispõe de um método com alta sensibilidade. Tem-se utilizado o xenodiagnóstico $0^{9}$, provavelmente pela sua melhor aplicabilidade em grandes populações e menor exigência tecnológica. Foi introduzido como método de investigação parasitológica da infecção chagásica por Brumpt ${ }^{7} \mathrm{em} \mathrm{1914,}$ e ao longo dos anos tem sido empregado em trabalhos de campo, envolvendo dezenas de pacientes de uma só vez ${ }^{9} 131920$, em estudos da sensibilidade das diferentes espécies de triatomíneos à infecção pelo $T$. cruzi $i^{3} 456212230$, no controle de cura 81125 e na indicaçāo da parasitemia em humanos 101728 ou em animais de experimentação 29 .

1. Departamento de Medicina Tropical do Instituto Oswaldo Cruz, Caixa Postal 926, Rio de Janeiro.

2. Núcleo de Medicina Tropical da Universidade Federal da Paraíba, João Pessoa.

Recebido para publicação em 3/8/88.
Apesar dos esforços de alguns estudio$\operatorname{sos}^{2} 1415162124$, ainda não se tem uma padronização universalmente aceita para o xenodiagnóstico, provavelmente em função das muitas variáveis que estão envolvidas principalmente em sua aplicação e leitura. Como conseqüência, são praticamente impossiveis as comparações dos resultados existentes na literatura, tendo ęm vista os diferentes números, estádios e espécies de ninfas empregados e as diferentes técnicas utilizadas na identificação da infecção dos insetos.

O atual trabalho, parte de um amplo estudo préterapêutico específico da infecção chagásica crônica, tem como principal objetivo avaliar a parasitemia dos pacientes através do índice de triatomineos infectados em uma série de três xenodiagnósticos.

\section{MATERIAL E MÉTODOS}

Pacientes. Foram examinados 206 chagásicos crônicos (definidos sorologicamente pelas reaçooes de imunofluorescência indireta e fixação do complemento) do municipio de Virgem da Lapa, Minas Gerais, sendo 85 homens e 121 mulheres, com idades de 7 a 80 anos (média de 37,4 $\pm 14,6$ ), 96 não-cardiopatas e 110 cardiopatas (definidos clínica e eletrocardiograficamente em exame anterior). 
Pereira JB, Willcox HPF, Marcondes CB, Coura JR. Parasitemia em pacientes chagásicos crônicos avaliada pelo indice de triatomíneos infectados no xenodiagnóstico. Revista da Sociedade Brasileira de Medicina Tropical 22: 39-44, jan-mar, 1989.

Xenodiagnóstico. Cada paciente foi submetido a uma série de três exames: o $1{ }^{\circ}$ e o $2{ }^{\circ}$, respectivamente, em janeiro e julho de 1986 e o $3 \circ$ em janeiro de 1987. Os exames consistiram em aplicar 40 ninfas de 30 ou 4\% estádios de Triatoma infestans, distribuidas em 4 caixas, as quais eram colocadas 2 em cada antebraço, contendo cada uma 10 exemplares, por um tempo médio de 30 minutos. A verificação da infecção dos insetos foi feita decorridos 30 a 40 dias do repasto, através da observação microscópica das fezes obtidas por compressão abdominal de cada exemplar (leitura por inseto). As fezes eram obtidas diretamente sobre lâminas, adicionando-se tantas gotas de PBS pH 7.2 quantas fossem necessárias para tornar o material passivel de boa visualização. Examinava-se todo o material obtido nos casos negativos. Nào foram examinadas as ninfas encontradas mortas que, em geral, estavam secas. As ninfas empregadas no presente estudo foram fornecidas pelos insectários do Departamento de Medicina Tropical do Instituto Oswaldo Cruz e do Núcleo de Medicina Tropical da Universidade Federal da Paraiba.

Parasitemia. Tomamos como base o trabalho de Castro $^{9}$ e adotamos os seguintes critérios para a parasitemia: não detectada (quando todas as ninfas examinadas eram negativas); baixa (quando o percentual de ninfas positivas era maior que zero e menor ou igual a 2,0); média (quando esse percentual era maior que 2,0 e menor ou igual a 7) e alta (quando maior que $7,0)$.

$\mathrm{Na}$ análise estatística dos dados empregamos o teste qui-quadrado, considerando o nivel de significância para $\mathrm{p} \leq 0,05$.

\section{RESULTADOS}

Dos 206 pacientes examinados, $105(51,0 \%)$ apresentaram os três xenos negativos, $49(23,8 \%)$ apresentaram um xeno positivo, $27(13,1 \%)$ dois xenos positivos e $25(12,1 \%)$ os três xenos positivos. Do total de 618 xenos aplicados em todo o grupo, 178 $(28,8 \%)$ foram positivos e das 22.807 ninfas examinadas, $428(1,9 \%)$ estavam infectadas (distribuidas de acordo com o grupo etário na Tabela 1 ). Verificou-se o maior índice de ninfas positivas no grupo de pacientes de 7 a 9 anos e o menor no grupo de 30 a 39 anos.

Dentro dos critérios adotados, a parasitemia não foi detectada em $105(51,0 \%)$ pacientes, foi baixa em $55(26,7 \%)$, média em $27(13,1 \%)$ e alta em 19 $(9,2 \%)$. Na Tabela 2 estão distribuidos os niveis de

Tabela 1 - Percentual de triatomíneos positivos de acordo com o sexo e grupo etário dos pacientes.

\begin{tabular}{|c|c|c|c|c|c|c|c|c|c|}
\hline \multirow{2}{*}{$\begin{array}{l}\text { Grupo } \\
\text { etário } \\
\text { (anos) }\end{array}$} & \multicolumn{3}{|c|}{ Pacientes masculinos (85) } & \multicolumn{3}{|c|}{ Pacientes femininos (121) } & \multicolumn{3}{|c|}{ Total de Pacientes (206) } \\
\hline & $\begin{array}{c}\text { Triato- } \\
\text { mineos } \\
\text { exami- } \\
\text { nados }\end{array}$ & $\begin{array}{c}\text { Triato- } \\
\text { míneos } \\
\text { posi- } \\
\text { tivos }\end{array}$ & $\%$ & $\begin{array}{l}\text { Triato- } \\
\text { míneos } \\
\text { exami- } \\
\text { nados }\end{array}$ & $\begin{array}{c}\text { Triato- } \\
\text { míneos } \\
\text { posi- } \\
\text { tivos }\end{array}$ & $\%$ & $\begin{array}{l}\text { Triato } \\
\text { míneos } \\
\text { exami- } \\
\text { nados }\end{array}$ & $\begin{array}{l}\text { Triato- } \\
\text { míneos } \\
\text { Posi- } \\
\text { tivos }\end{array}$ & $\%$ \\
\hline 7- 9 & 441 & 14 & 3,2 & 785 & 31 & 4,0 & 1226 & 45 & 3,7 \\
\hline $10-19$ & 1075 & 9 & 0,8 & 1311 & 30 & 2,3 & 2386 & 39 & 1,6 \\
\hline $20-29$ & 972 & 8 & 0,8 & 3055 & 67 & 2,2 & 4027 & 75 & 1,9 \\
\hline $30-39$ & 1773 & 5 & 0,3 & 3365 & 39 & 1,2 & 5138 & 44 & 0,9 \\
\hline $40-49$ & 2225 & 62 & 2,8 & 2230 & 46 & 2,1 & 4455 & 108 & 2,4 \\
\hline $50-59$ & 2200 & 52 & 2,3 & 1899 & 42 & 2,2 & 4119 & 94 & 2,3 \\
\hline$\geq 60$ & 671 & 15 & 2,2 & 785 & 8 & 1,0 & 1456 & 23 & 1,6 \\
\hline Total & 9.377 & 165 & 1,8 & 13.430 & 263 & 2,0 & 22.807 & 428 & 1,9 \\
\hline
\end{tabular}

Tabela 2 - Parasitemia em chagásicos crónicos de acordo com o sexo.

\begin{tabular}{|c|c|c|c|c|c|c|c|c|c|}
\hline \multirow[t]{2}{*}{$\overline{\text { Sexo }}$} & \multirow[t]{2}{*}{$\begin{array}{c}\text { Pacientes } \\
\text { examinados }\end{array}$} & \multicolumn{2}{|c|}{$\begin{array}{r}\text { Parasitemia } \\
\text { não detectada }\end{array}$} & \multicolumn{2}{|c|}{$\begin{array}{c}\text { Parasitemia } \\
\text { baixa }\end{array}$} & \multicolumn{2}{|c|}{$\begin{array}{l}\text { Parasitemia } \\
\text { média }\end{array}$} & \multicolumn{2}{|c|}{$\begin{array}{c}\text { Parasitemia } \\
\text { alta }\end{array}$} \\
\hline & & Pacientes & $\%$ & Pacientes & $\%$ & Pacientes & $\%$ & Pacientes & $\%$ \\
\hline Masculino* & 85 & 49 & 57,6 & 18 & 21,2 & 9 & 10,6 & 9 & 10,6 \\
\hline Feminino * & 121 & 56 & 46,3 & 37 & 30,6 & 18 & 14,9 & 10 & 8,3 \\
\hline Total & 206 & 105 & 51,0 & 55 & 26,7 & 27 & 13,1 & 19 & 9,2 \\
\hline
\end{tabular}

* $p>0,05$ 
Pereira JB, Willcox HPF, Marcondes CB, Coura JR. Parasitemia em pacientes chagásicos crônicos avaliada pelo indice de triatomineos infectados no xenodiagnóstico. Revista da Sociedade Brasileira de Medicina Tropical 22: 39-44, jan-mar, 1989.

parasitemia de acordo com o sexo dos pacientes, sem diferença significativa. $\mathrm{Na}$ Tabela 3 assinalamos a distribuição em relação aos grupos etários, destacando a maior freqüência de parasitemia não detectada no grupo de 20 a 39 anos e da parasitemia baixa no grupo de 60 anos ou mais.
$\mathrm{Na}$ Tabela 4 estão assinaladas as freqüências dos níveis da parasitemia em relação à presença ou não de cardiopatia, com destaque para a maior ocorrência de parasitemia alta entre os pacientes com cardiopatia.

$\mathrm{Na}$ Tabela 5 registramos os pacientes com parasitemia alta. Todos apresentaram os três xenos

Tabela 3 - Parasitemia em chagásicos crônicos de acordo com o grupo etário.

\begin{tabular}{|c|c|c|c|c|c|c|c|c|c|}
\hline \multirow{2}{*}{$\begin{array}{l}\text { Grupo } \\
\text { etário } \\
\text { (anos) }\end{array}$} & \multirow[t]{2}{*}{$\begin{array}{c}\text { Pacientes } \\
\text { Examinados }\end{array}$} & \multicolumn{2}{|c|}{$\begin{array}{c}\text { Parasitemia } \\
\text { Não detectada }\end{array}$} & \multicolumn{2}{|c|}{$\begin{array}{l}\text { Parasitemia } \\
\text { baixa }\end{array}$} & \multicolumn{2}{|c|}{$\begin{array}{l}\text { Parasitemia } \\
\text { média }\end{array}$} & \multicolumn{2}{|c|}{$\begin{array}{l}\text { Parasitemia } \\
\text { alta }\end{array}$} \\
\hline & & Pacientes & $\%$ & Pacientes & $\%$ & Pacientes & $\%$ & Pacientes & $\%$ \\
\hline $7-19$ & 33 & 13 & 39,4 & 11 & 33,3 & 6 & 18,2 & 3 & 9,1 \\
\hline $20-39$ & 83 & 51 & 61,4 & 16 & 19,3 & 11 & 13,3 & 5 & 6,0 \\
\hline $40-59$ & 77 & 37 & 48,0 & 20 & 26.0 & 10 & 13,0 & 10 & 13,0 \\
\hline$\geqslant 60$ & 13 & 4 & 30,8 & 8 & 61,5 & 0 & 0,0 & 1 & 7,7 \\
\hline Total & 206 & 105 & 51,0 & 55 & 26,7 & 27 & 13,1 & 19 & 9,2 \\
\hline
\end{tabular}

Tabela 4 - Parasitemia em chagásicos crônicos de acordo com a presença ou não de cardiopatia.

\begin{tabular}{cccccc}
\hline Cardiopatia & Pacientes & Parasitemià & Parasitemia & Parasitemia & Parasitemia \\
Examinados & $\begin{array}{c}\text { Não detectada } \\
\text { Pacientes \% }\end{array}$ & $\begin{array}{c}\text { baixa } \\
\text { Pacientes \% }\end{array}$ & $\begin{array}{c}\text { média } \\
\text { Pacientes \% }\end{array}$ & $\begin{array}{c}\text { alta } \\
\text { Pacientes \% }\end{array}$
\end{tabular}

\begin{tabular}{lrrrrrrrrr}
\hline Ausente & 96 & 52 & 54,2 & 31 & 32,3 & 9 & 9,4 & 4 & 4,1 \\
Presente & 110 & 53 & 48,2 & 24 & 21,8 & 18 & 16,4 & 15 & 13,6 \\
\hline Total & 206 & 105 & 51,0 & 55 & 26,7 & 27 & 13,1 & 19 & 9,2 \\
\hline & & $p>0,05$ & $p>0,05$ & $p>0,05$ & & $p<0,05$
\end{tabular}

Tabela 5 - Pacientes chagásicos crônicos com parasitemia alta.

\begin{tabular}{|c|c|c|c|c|c|c|c|c|c|c|}
\hline № & Registro & Idade & Sexo & Cardiopatis & a Eletrocardiograma & 1.9 xeno* & 20 xeno & $3 \%$ xeno & Total & $(\%)$ \\
\hline 01 & VLA 22 & 8 & $\mathrm{~m}$ & Presente & Bloqueio auriculo-ventricular II & $6: 37$ & $1: 33$ & 1:39 & $8: 109$ & 7,3 \\
\hline 02 & VLA 44 & 7 & $\mathrm{f}$ & Ausente & Normal & $11: 37$ & $4: 37$ & $5: 39$ & $20: 113$ & 17,7 \\
\hline 03 & VLB 68 & 14 & $\mathrm{f}$ & Ausente & Normal & $2: 28$ & $2: 35$ & $4: 32$ & $8: 95$ & 8,4 \\
\hline 04 & VLC 16 & 21 & f & Presente & Extrassistole ventricular isolada & $2: 39$ & $4: 35$ & $3: 38$ & $9: 112$ & 8,0 \\
\hline 05 & VLC 30 & 21 & f & Presente & Taquicardia sinusal + alt. prim. repolar. ventric. & $2: 34$ & $7: 36$ & 3:39 & $12: 109$ & 11,0 \\
\hline 06 & VLC 87 & 25 & $\mathrm{f}$ & Presente & BRD III + HBAE & $3: 37$ & $6: 39$ & $2: 36$ & $11: 112$ & 9,8 \\
\hline 07 & VLD 12 & 38 & f & Presente & BRD III + HBAE & $6: 39$ & $2: 40$ & $3: 36$ & $11: 115$ & 9,6 \\
\hline 08 & VLD 79 & 35 & $\mathrm{f}$ & Ausente & Normal & $1: 37$ & $3: 32$ & $4: 37$ & $8: 106$ & 7,5 \\
\hline 09 & VLE 80 & 47 & $\mathrm{~m}$ & Ausente & Normal & $4: 38$ & $6: 35$ & $4: 40$ & $14: 113$ & 12,4 \\
\hline 10 & VLE 88 & 43 & $\mathrm{~m}$ & Presente & BRD III + HBAE & $4: 40$ & $1: 37$ & $3: 33$ & $8: 110$ & 7,3 \\
\hline 11 & VLE 95 & 46 & $\mathrm{f}$ & Presente & Extrassistoles ventricular freqüente & $3: 37$ & $1: 39$ & $9: 39$ & $13: 115$ & 11,3 \\
\hline 12 & VLE 99 & 49 & $\mathrm{~m}$ & Presente & $\mathrm{BRD} \mathrm{III}+\mathrm{HBAE}$ & $3: 40$ & $5: 38$ & $4: 37$ & $12: 115$ & 10,4 \\
\hline 13 & VLE 103 & 40 & $\mathbf{m}$ & Presente & Alteraẹ̃ão primária da repolarização ventricular & $6: 37$ & $8: 40$ & $3: 40$ & $17: 117$ & 14,5 \\
\hline 14 r & VLE 107 & 40 & $\mathrm{f}$ & Presente & Bradicardia sinual + alt. prim. repolar. ventric. & $2: 38$ & $10: 36$ & $4: 40$ & $16: 114$ & 14,0 \\
\hline 15 & VLF 36 & 53 & $\mathrm{~m}$ & Presente & Bloqueio auriculo-ventricular II & $1: 35$ & $7: 38$ & $2: 35$ & $10: 108$ & 9,2 \\
\hline 16 & VLF 44 & 54 & $\mathrm{f}$ & Presente & Extrassistole ventricular isolada & $15: 37$ & $2: 32$ & $4: 36$ & $21: 105$ & 20,0 \\
\hline 17 & VLF 67 & 51 & $\mathbf{m}$ & Presente & BRE III + Extrassístoles ventricular freqüente & $1: 35$ & $1: 38$ & $6: 38$ & 8:111 & 7,2 \\
\hline 18 & VLF 74 & 57 & $\mathbf{m}$ & Presente & BRD III + Zona eletricamente inativa & $2: 37$ & $5: 35$ & $3: 40$ & $10: 112$ & 9,0 \\
\hline 19 & VLG 67 & 60 & $\mathbf{m}$ & Presente & Bloqueio auriculo-ventricular II & $5: 38$ & $4: 39$ & $2: 40$ & $11: 117$ & 9,4 \\
\hline
\end{tabular}

- No de triatomíneos positivos: No de triatomíneos examinados.

BRD III = bloqueio completo do ramo direito; HBAE = hemibloqueio anterior esquerdo; BRE III = bloqueio completo do ramo esquerdo 
Pereira JB, Willcox HPF, Marcondes CB, Coura JR. Parasitemia em pacientes chagásicos crônicos avaliada pelo índice de triatomíneos infectados no xenodiagnóstico. Revista da Sociedade Brasileira de Medicina Tropical 22: 39-44, jan-mar, 1989.

positivos, indicando com isso uma importante associação entre esse nivel de parasitemia e a persistência dos xenos positivos. Por outro lado, somente $22.2 \%$ dos pacientes com parasitemia média apresentaram essa persistência da xenopositividade e nenhum caso foi assinalado entre os pacientes com parasitemia baixa.

\section{DISCUSSÃO}

Empregamos o xenodiagnóstico para a avaliação da parasitemia, pela sua vantajosa aplicabilidade em grande número de pessoas especialmente em áreas endêmicas, além do seu melhor rendimento em relação aos demais métodos, até o momento, indicados para o diagnóstico parasitológico na fase crônica da infecção chagásica 1223 . Utilizamos o $T$. infestans por ser a espécie que dispúnhamos em quantidade suficiente ao desenvolvimento do trabalho, apesar da existência de estudos que revelam seu menor rendimento quando comparado com o Dipetalogaster maximus 49 e com - Panstrongylus megistus 62122 . Examinamos as fezes obtidas por compressão abdominal, ao invés de retirarmos o aparelho digestivo dos insetos porque desejávamos mantê-los vivos para outras investigações, embora existam estudos que revelam sua menor eficiência na identificação da infecção 1621 . Houve um índice de mortalidade de ninfas de 5,0\%, que acreditamos não ter influído no resultado global.

A análise da parasitemia, no presente trabalho, tomando como unidade de observação o inseto, ao invés do "pool" de insetos como fez Castro", deve-se ao fato de que o inseto representa com maior fidelidade o grau de parasitemia do que o "pool", na medida em que este pode conter mais de um inseto positivo, subdimensionando o resultado, especialmente, nos casos com parasitemia baixa ou até média. $O$ mesmo não deve ocorrer com os casos de parasitemia alta, que por apresentarem um grande número de insetos positivos, em obediência às leis do acaso, todos os "pools" poderão ser positivos.

Consideramos a expressão parasitemia não detectada, ao invés de parasitemia negativa, por acreditarmos que a sensibilidade do xenodiagnóstico na fase crônica não nos autoriza a afirmar que pacientes xenonegativos não apresentem parasitas circulantes.

O número de pacientes com um ou mais xenos, positivos, no presente trabalho, foi de $101(49,0 \%)$ o qual está dentro do esperado para os pacientes da área em questão, tendo em vista que Coura e cols ${ }^{13}$ e Pereira e Coura 19 em trabalhos anteriores na mesma região, respectivamente, há dez e seis anos, aplicando somente um xeno por paciente, observaram os índices de $24,49 \%$ e $36,7 \%$. Atribuimos à repetição dos exames, uma ampliação do índice de pacientes xenopositivos 10 em nosso estudo.
Consideramos o percentual global de $1,9 \%$ de ninfas infectadas como baixo, contudo pode ter sido influenciado pela espécie de triatomíneo empregada, pela técnica utilizada na identificação da infecção, pela idade média do grupo de pacientes em tomo da quarta década da vida, na qual observa-se o menor indice de ninfas infectadas (Tabela 1) e pela interrupção da transmissão da infecção por triatomíneos na área, responsável pela redução de casos recentes, os quais apresentam o maior índice de ninfas infectadas (Tabela 1).

Os nossos resultados sobre a parasitemia podem ser confrontados com os obtidos por Castro ${ }^{9} \mathrm{em}$ Mambai-GO, que na ocasião aplicou três xenos em cada paciente por um periodo inferior a dois anos. Esse autor assinalou parasitemia baixa em 197 (67,5\%) pacientes, dos quais $92(31,5 \%)$ eram xenonegativos e $105(35,9 \%)$ eram xenopositivos; parasitemia média em $68(23,3 \%)$ e alta em $27(9,2 \%)$. Esses dados revelam semelhança na freqüência de pacientes com parasitemia alta em ambos os estudos, embora Castro tenha utilizado o "pool", em geral de cinco ninfas, como unidade de observação. Isto reforça a idéia de que para esse tipo de parasitemia ambos os métodos de análise, fezes de um inseto e "pool" de fezes, serão capazes de dimensioná-la adequadamente.

Não haveria diferença entre as frequêencias de pacientes com parasitemia baixa em ambos os trabalhos se considerássemos, nesse grupo, os pacientes com parasitemia não detectada. No entanto, houve diferença significativa nas freqüèncias de pacientes com parasitemia média que acreditamos depender das amostras.

A principal divergência, entre o atual trabalho e o de Castro ${ }^{9}$, está no fato de que observamos um maior indice de párasitemia alta nos pacientes cardiopatas, enquanto esse autor não encontrou influência da parasitemia na doença de Chagas crônica. Acreditamos que ambos os resultados estão coerentes com os grupos de pacientes estudados. Em nossa casuística, $16 \%$ dos pacientes tinham menos de 20 anos, enquanto na de Mambai este percentual era de 35\%, diferença importante, na medida em que se sabe que os indices de doença nesse grupo de pacientes são menores em relação aos demais idosos, no entanto, são maiores os índices de xenopositivos e de ninfas infectadas (Tabela 1).

Foi comum a ambos os trabalhos a predominância de pacientes com parasitemia baixa, em relaçāo aos de média e alta, indicando desse modo ser uma característica da fase crônica da infecção chagásica. Também foram semelhantes as freqüências de pacientes com parasitemia persistente, ou seja, com todos os xenos positivos. Este achado esteve ligado à totalidade dos pacientes com parasitemia alta e a um quinto dos pacientes com parasitemia média. Não dispomos de 
Pereira JB, Willcox HPF, Marcondes CB, Coura JR. Parasitemia em pacientes chagásicos crónicos avaliada pelo indice de triatomíneos infectados no xenodiagnóstico. Revista da Sociedade Brasileira de Medicina Tropical 22: 39-44, jan-mar 1989.

explicação consistente para justificar a existência da parasitemia persistente em pacientes de ambos os sexos e nos diferentes grupos etários. Esses pacientes representam um percentual em torno de $12 \%$. Cabenos questionar os possiveis fatores determinantes desse evento. Seriam esses pacientes portadores de distúrbios nos mecanismos de defesa capazes de "permitirem" a evasão dos parasitas? Ou estaríamos diante de parasitas com propriedades intrínsecas capazes de lhes permitirem o "escape" até mesmo dos mecanismos normais de defesa desses pacientes? Ou ambos os fenômenos estariam envolvidos? Advinda desses ou de outros mecanismos a resposta para explicar a parasitemia persistente, caberão ainda as questōes relativas às repercussões clínico-evolutivas dessa persistência, que, elucidadas, nos permitirão estabelecer com segurança os benefícios para o paciente, ao instituirmos a terapeutica especifica.

\section{SUMMARY}

As part of a pre-treatment study of chronic Chagas infections, the parasitemia of 206 patients ( 85 men and 121 women, aged 7 to 80 y) from Virgem da Lapa, Minas Gerais State Brazil, was evaluated by three xenodiagnoses per patient during a one year period. Each time, 40 3rd or 4th instar nymphs of Triatoma infestans were applied. The parasitemia was arbitrarily classified as: not detected (when all nymphs were negative), low (when the number of infected nymphs was less than $2 \%$ ), medium (when it was higher than $2 \%$ and up to $7 \%$ ) and high (when higher than 7\%). The parasitemia was not detected in $105(51 \%)$ of the patients, and was considered low in $55(26,7 \%)$, medium in $27(13.1 \%)$ and high in 19 (9,2\%). There was no significant differences in levels of parasitemia in relation to sex or age, but the high parasitemia was more frequent among the patients with chagasic cardiomyopathy. Persistent parasitemia (all three xenodiagnoses positive) was observed in $100 \%$ of the patients with high parasitemia, in $22.2 \%$ with medium and in none with low parasitemia.

Key-words: Chagas' disease. Xenodiagnosis. Parasitemia. Triatoma infestans.

\section{AGRADECIMENTOS}

Ao Dr. Leônidas Deane pela revisão do texto e sugestōes. A Angela Cristina Veríssimo Junqueira, Júlio Cesar Miguel, Nelmo Bruno, Joaquim Mendes e Zulmira Rios pela colaboração na leitura dos xenos.

\section{REFERENNCIAS BIBLIOGRÁFICAS}

1. Albuquerque RDR, Fernandes LAR, Funayama GK, Ferriolli Filho F, Siqueira AF. Hemoculturas seriadas com o meio de Warren em pacientes com reação de

- Guerreiro Machado positiva. Revista do Instituto de Medicina Tropical de São Paulo 14: 1-5, 1972.

2. Almeida SP, Miles MA, Marsden PD. Verificação da susceptibilidade à infecçâo por Trypanosoma cruzi, dos estágios evolutivos de Rhodnius neglectus. Revista Brasileira de Biologia 33: 43-52, 1973.

3. Almeida SP, Sherlock IA, Fahel E. Novo procedimento do xenodiagnóstico na forma crônica da doença de i Chagas. Memórias do Instituto Oswaldo Cruz 74: 285 288, 1976.

4. Alvarenga NJ, Bronfen E. Interação do Trypanosoma cruzi com diferentes vetores: uso para o xenodiagnóstico. Revista da Sociedade Brasileira de Medicina Tropical 17: 145-149, 1984.

5. Alvarenga NJ, Cuba CC, Barreto AC, Marsden PD, Macedo V. Valor comparativo entre Dipetalogaster maximus e Triatoma infestans no diagnóstico parasitológico de pacientes chagásicos crônicos com sorologia positiva. In: Resumos do XIII Congresso da Sociedade Brasileira de Medicina Tropical e II Congresso da Sociedade Brasileira de Parasitologia, Brasília, Distrito Federal, 1977.

6. Bronfen E, Dias JCP, Gouveia SC. Infecção experimental de Triatoma infestans e Panstrongylus megistus pela cepa Y do Trypanosoma cruzi (Silva e Nussenzweig, 1953). Revista de Patologia Tropical 13: 1-7, 1984.

7. Brumpt E. O xenodiagnóstico. Aplicação ao diagnóstico de algumas infecçōes parasitárias e em particular à tripanosomose de Chagas. Anais Paulista de Medicina e Cirurgia 3: 97-102, 1914.

8. Cançado JR, Barra UD, Mourão OG, Alvares JM, Oliveira JPM, Machado JR, Salgado AA. Bases para a avaliação do tratamento especifico da doença de Chagas humana segundo a parasitemia. Revista da Sociedade Brasileira de Medicina Tropical 7: 155-166, 1973.

9. Castro CN. Influência da parasitemia no quadro clínico da doença de Chagas. Revista de Patologia Tropical 9: 73-136, 1980.

10. Castro CN, Alves MT, Macêdo VO. Importância da repetição do xenodiagnóstico para avaliação da parasitemia na fase crônica da doença de Chagas. Revista da Sociedade Brasileira de Medicina Tropical 16: 98-103, 1983.

11. Cerisola JA, Neves da Silva N, Prata A, Shenone H, Rohwedder R. Evalución mediante xenodiagnóstico de la efectividad del nifurtimox en la infección chagásica crónica humana. Boletin Chileno de Parasitologia 32: 51-62, 1977.

12. Chiari E, Brener Z. Contribuição ao diagnóstico parasitológico da doença de Chagas na sua fase crônica. Revista do Instituto de Medicina Tropical de São Paulo 8: 134-138, 1966.

13. Coura JR, Abreu LL, Dubois LEG, Correia-Lima F, Arruda Junior E, Willcox HPF, Anunziato N, Petana W. Morbidade da doença de Chagas. II - Estudos iseccionais em quatro áreas de campo no Brasil. Memórias do Instituto Oswaldo Cruz 79: 101-124, 1984. 
Pereira JB, Willcox HPF, Marcondes CB, Coura JR. Parasitemia em pacientes chagásicos crônicos avaliada pelo indice de triatomineos infectados no xenodiagnóstico. Revista da Sociedade Brasileira de Medicina Tropical 22: 39-44, jan-mar, 1989.

14. Cuba CC, Alvarenga NJ, Barreto AC, Marsden PD, Macedo V, Gama MP. Dipetalogaster maximus (Hemiptera,Triatominae) for xenodiagnosis of patients with serologically detectable Trypanosoma cruzi infection. Transactions of the Royal Society of Tropical Medicine and Hygiene 73: 524-527, 1979.

15. Dias E. Técnica do xenodiagnóstico na moléstia de Chagas. Memórias do Instituto Oswaldo Cruz 35: 335$345,1940$.

16. Guedes AS. Determinação do indice de infecção de triatomineos por Schizotrypanum cruzi pelo exame simples das fezes obtidas por expressão e por dissecação do intestino posterior do inseto. Dados preliminares. Revista Brasileira de Malariologia e Doenças Tropicais 4: 464-465, 1952.

17. Marsden PD, Mott KE, Prata A. The prevalence of Trypanosoma cruzi parasitaemia in 8 families in an endemic area. Gazeta Médica da Bahia 69:65-69, 1969.

18. Neal RA, Miles RA. The sensitivity of culture methods to detect experimental infections of Trypanosoma cruzi and comparison with xenodiagnosis. Revista do Instituto de Medicina Tropical de São Paulo 19: 170-176, 1977.

19. Pereira JB, Coura JR. Morbidade da doença de Chagas. Estudo seccional em uma área endêmica, Virgem da Lapa, Minas Gerais. Revista da Sociedade Brasileira de Medicina Tropical 19: 139-148, 1986.

20. Pereira JB, Coura JR. Morbidade da doença de Chagas em populaçōes urbanas do Sertão da Paraiba. Revista da

4 Sociedade Brasileira de Medicina Tropical 20: 101-107, 1987.

21. Perlowagora-Szumlevicz A, Muller AC. Experiments in a search for an insect model for xenodiagnosis. I - The prevalence and intensity of infection with $T$. cruzi in nine vectors species. In: Anais do Congresso Internacional sobre a doença de Chagas, Rio de Janeiro, p E 11-E 17, 1979.
22. Perlowagora-Szumlevicz A, Muller CA. Studies in search of a suitable experimental insect model for xenodiagnosis of hosts with Chagas' disease. 2 - Attempts to upgrade the reliability and the efficacy of xenodiagnosis in chronic Chagas' disease. Memórias do Instituto Oswaldo Cruz 82: 259-272, 1987.

23. Pifano CF. El diagnóstico parasitológico de la enfermedad de Chagas em fase crónica. Estudio comparativo entre la gota gruesa, el xenodiagnóstico, el hemocultivo y las inoculaciones experimentales em animales sensibles. Abstract In Tropical Disease Bulletin 52: 880-881, 1955.

24. Salgado AA. Consideraciones sobre metodologia y sensibilidad del xenodiagnóstico. Boletin Chileno de Parasitologia. 24: 9-13, 1969.

25. Salgado AA. Xenodiagnosis in the selection of patients and evaluation of treatment. In: American Trypanosomiasis Research, Belo Horizonte, p. 223, 1975.

26. Schenone $\mathrm{H}$, Alfaro $\mathrm{E}$, Reyes $\mathrm{H}$, Taucher $\mathrm{E}$. Valor del xenodiagnóstico en la infección chagásica crónica. Boletin Chileno de Parasitologia 23: 149-154, 1968.

27. Schenone H, Alfaro E, Rojas A. Bases y rendimento del xenodiagnóstico en la infección chagásica human. Boletín Chileno de Parasitologia 29: 24-26, 1974.

28. Schenone H, Rojo M, Rojas A, Concha L. Positividad diurna y nocturna del xenodiagnóstico en un paciente con infección chagásica crónica de parasitemia permanente. Boletin Chileno de Parasitologia 32: 63-66, 1977.

29. Sherlock IA. Parasitemia constante durante 24 horas consecutivas na infecção experimental pelo Trypanosoma cruzi. Revista da Sociedade Brasileira de Medicina Tropical 17: 137-144, 1984.

30. Sherlock IA, Almeida SP. Diferenças de susceptibilidade à infecção com $T$. cruzi entre espécies de triatomineos alimentados em cão, tatu e camundongo infectados. Revista da Sociedade Brasileira de Medicina Tropical 7: 87-98, 1971. 